\title{
Computer aided quantification of intratumoral stroma yields an independent prognosticator in rectal cancer
}

\author{
Oscar G. F. Geessink ${ }^{1,2,3}$ - Alexi Baidoshvili ${ }^{3}$ - Joost M. Klaase ${ }^{4}$ - Babak Ehteshami Bejnordi ${ }^{2}$ - Geert J. S. Litjens ${ }^{1,2}$. \\ Gabi W. van Pelt ${ }^{5}$. Wilma E. Mesker ${ }^{5}$. Iris D. Nagtegaal ${ }^{1}$. Francesco Ciompi ${ }^{1,2}$ • Jeroen A. W. M. van der Laak ${ }^{1,2,6}$
}

Accepted: 7 February 2019 / Published online: 1 March 2019

(C) The Author(s) 2019

\begin{abstract}
Purpose Tumor-stroma ratio (TSR) serves as an independent prognostic factor in colorectal cancer and other solid malignancies. The recent introduction of digital pathology in routine tissue diagnostics holds opportunities for automated TSR analysis. We investigated the potential of computer-aided quantification of intratumoral stroma in rectal cancer whole-slide images.

Methods Histological slides from 129 rectal adenocarcinoma patients were analyzed by two experts who selected a suitable stroma hot-spot and visually assessed TSR. A semi-automatic method based on deep learning was trained to segment all relevant tissue types in rectal cancer histology and subsequently applied to the hot-spots provided by the experts. Patients were assigned to a 'stroma-high' or 'stroma-low' group by both TSR methods (visual and automated). This allowed for prognostic comparison between the two methods in terms of disease-specific and disease-free survival times.

Results With stroma-low as baseline, automated TSR was found to be prognostic independent of age, gender, pT-stage, lymph node status, tumor grade, and whether adjuvant therapy was given, both for disease-specific survival (hazard ratio $=2.48(95 \%$ confidence interval 1.29-4.78)) and for disease-free survival (hazard ratio $=2.05(95 \%$ confidence interval 1.11-3.78)). Visually assessed TSR did not serve as an independent prognostic factor in multivariate analysis.

Conclusions This work shows that TSR is an independent prognosticator in rectal cancer when assessed automatically in userprovided stroma hot-spots. The deep learning-based technology presented here may be a significant aid to pathologists in routine diagnostics.
\end{abstract}

Keywords Rectal carcinoma $\cdot$ Tumor-stroma ratio $\cdot$ Prognosis $\cdot$ Computational pathology $\cdot$ Automated analysis $\cdot$ Deep learning

Authors Oscar G. F. Geessink and Alexi Baidoshvili contributed equally to this work.

Jeroen A. W. M. van der Laak

Jeroen.vanderlaak@ radboudumc.nl

1 Department of Pathology, Radboud Institute for Health Sciences, Radboud University Medical Center, P.O.Box 9101, 6500 HB Nijmegen, The Netherlands

2 Diagnostic Image Analysis Group (DIAG), Radboud University Medical Center, Nijmegen, The Netherlands

3 Laboratory for Pathology East Netherlands (LabPON), Hengelo, The Netherlands

4 Department of Surgery, Medisch Spectrum Twente, Enschede, The Netherlands

5 Department of Surgery, Leiden University Medical Center, Leiden, The Netherlands

6 Center for Medical Image Science and Visualization, Linköping University, Linköping, Sweden

\section{Introduction}

In most solid malignancies, therapeutic decision making is primarily based on pathological staging of tumors. The traditional tumor, (lymph) node, metastasis (TNM) staging system [1] is routinely used to estimate patient prognosis and guide treatment worldwide. For certain tumor types, however, the TNM system lacks accuracy in assessing the metastatic potential of a tumor. For instance, TNM stage II colorectal cancer (CRC) comprises a heterogeneous group with a diverse outcome [2]. As a result, the TNM stage is not informative for therapy planning of these patients, leading to both under- and over-treatment. Reliable new biomarkers are needed to guide personalized adjuvant treatment for these groups of patients.

A widely studied prognostic factor is the tumor-stroma ratio (TSR), expressing the relative amounts of tumor and intratumoral stroma. TSR is a straightforward measure which can be assessed by microscopic inspection of hematoxylin and 
eosin (H\&E) stained tissue sections. TSR has been shown to yield prognostic information in a range of solid malignancies, including breast cancer [3-5] and lung cancer [6, 7]. Generally, TSR is an independent prognostic factor, where a high content of intratumoral stroma is associated with a poor prognosis. A number of previous studies showed promising results on the prognostic relevance of TSR in CRC [8-12]. Despite this evidence, there is no implementation in routine pathology reporting. This may be attributed to the variety in methodology and the lack of a standardized procedure for TSR assessment. Published studies propose visual assessment ('eyeballing'), systematic point counting, and the use of scanned (digitized) tissue sections (whole slide images; WSI). Although good inter-observer agreement was found in earlier studies [9, 11, 13], visual assessment of pathological quantitative features in general may suffer from reproducibility issues.

To facilitate an objective and standardized TSR assessment, image analysis and machine learning algorithms have been applied on H\&E-stained sections of CRC before, however, these algorithms were applied to image regions extracted from WSI. Computer-aided tumor and stroma quantification has been proposed based on automated tissue segmentation in $\mathrm{H} \&$ E-stained sections using a combination of hand-crafted features and machine learning [14]. Furthermore, TSR has been computed via automated point counting in H\&E-stained images [15]. Similar image analysis techniques based on classical machine learning have been applied to tissue microarrays for epidermal growth factor receptor (EGFR) detection by immunohistochemistry $[16,17]$. A new branch of machine learning algorithms, so-called deep learning algorithms, have recently entered the field of computational pathology and shown promise for automating certain tasks in histopathology. Detection of sentinel lymph node metastases [18] and of cancer in prostate biopsies [19] could successfully be performed using convolutional neural networks (CNN), a specific type of deep learning. We recently showed [20] that a deep learning-based algorithm can distinguish between 9 different types of tissue in CRC WSI with an overall accuracy of $93.8 \%$.

The present study aims to leverage our previously developed CNN for automated TSR assessment in the CRC subclass of rectal adenocarcinomas. Only a limited number of studies have been published on TSR for rectal cancers and in a sub-analysis $(n=43)$ by West et al. [12] its prognostic value could not be confirmed. Work by Scheer et al. [8] recently showed that TSR has potential as a prognostic factor for survival in surgically treated rectal cancer patients, however, TSR was only found to be an independent prognosticator in lymph node metastasis negative cases. The performance of the automated TSR system described here will be compared with data from human experts and its prognostic value will be evaluated in terms of disease-specific and disease-free survival times.

\section{Materials and methods}

\subsection{Patients}

An existing cohort of 154 patients [8] with rectal adenocarcinoma stages I-III was used. All patients received curative surgery in the period 1996-2006 at the Medisch Spectrum Twente hospital (The Netherlands). No patient was neoadjuvantly treated with radiotherapy and/or chemotherapy or died within 30 days after surgery. At the time of surgery, none of the patients had known distant metastases, inflammatory bowel disease, hereditary nonpolyposis colorectal cancer (HNPCC) or other/earlier cancers. Histopathological data were obtained from the Laboratory for Pathology Eastern Netherlands (LabPON). Clinical data were obtained from the Medisch Spectrum Twente hospital and the Netherlands Comprehensive Cancer Organization (IKNL). Collected clinicopathological data included tumor grade (differentiation), depth of invasion (pT) and lymph node involvement $(\mathrm{pN})$ according to the Union Internationale Contre le Cancer/American Joint Cancer Committee (UICC/AJCC) TNM staging system [1]. Data regarding adjuvant therapy and local or distant recurrence were also available.

\subsection{Tissue slide preparation and scanning}

According to standard procedures at LabPON, formalin fixed and paraffin embedded tissue sections were cut at $2 \mu \mathrm{m}$ and stained in an automatic stainer with hematoxylin and eosin (H\&E) for routine diagnostic purposes. For the present study, a single slide per patient was selected which contained the most invasive part of the tumor and was used in diagnostics to assess the tumor pT-status. Slides were scanned at $\times 200$ total magnification (tissue level pixel size $\sim 0.455 \mu \mathrm{m} /$ pixel) using a Hamamatsu NanoZoomer 2.0-HT (C9600-13) scanner (Herrsching, Germany).

\subsection{Visual estimation of intratumoral stroma}

Two observers (GvP, WM; both > 10 years of experience with TSR scoring) independently scored the slides using a conventional light microscope according to a previously published protocol for TSR assessment $[9,10]$. Briefly, the procedure consisted of 1) coarse localization of the tissue area with the highest intratumoral stroma content at low microscope magnification, and 2) selection of one field of view at $\times 100$ total magnification and visual estimation of the tumor-stroma ratio (TSR-visual) in the selected circular region. Ideally, the selected region should meet the following criteria: high intratumoral stroma content (predominantly found at the invasive margin of a tumor); presence of tumor cells at all borders of the field of view; no large quantities of muscle, mucus, necrosis or large vessels; and no tears or tissue retraction artefacts. As much as possible, the region with the highest stroma 
content (stroma hot-spot) was selected that met all the above requirements. TSR-visual was estimated by both observers independently, using $10 \%$ increments. As a result of the specific microscope and lenses used, the specimen-level diameter of the circular region was $1.8 \mathrm{~mm}$ at $\times 100$ magnification. There is a lot of variation among published studies concerning used TSR procedures (e.g. major differences in the location and size of the assessed tissue regions as well as what was actually measured: relative tumor or stroma content). For clarity, in this study the tumor-stroma ratio was defined as TSR = $100 \% \times$ [intratumoral stroma area] / [tumor area + intratumoral stroma area]. Lumen, tears and other tissue types in the selected circular region were excluded during visual estimation. Lastly, the tissue region considered most suitable for TSR assessment was identified during a consensus meeting between the two observers in which 1) a binary TSR consensus score was determined: 'stroma-low' or 'stromahigh', and 2), the center of the stroma hot-spot was marked on the glass slide.

\subsection{Automated computation of intratumoral stroma}

To study the value of applying a deep learning algorithm for automated TSR assessment (TSR-auto), a CNN was developed similar to a previously published algorithm [20]. The CNN performs tissue segmentation (i.e. subdivision of tissue areas) of H\&E-stained rectal cancer WSI into nine different classes: tumor, intratumoral stroma, necrosis, muscle, healthy epithelium, fatty tissue, lymphocytes, mucus and erythrocytes. The CNN was trained using manually annotated regions in 74 WSI taken from the cohort used in this study. Regions to annotate were selected for covering tissue variety across WSI, rather than producing exhaustive annotations on a small number of WSI. Annotations were produced by a pathology researcher (OG) and a medical student, and were checked and corrected when deemed necessary by an experienced pathologist (AB). A digital staining normalization method [21] was applied to all WSI as a pre-processing step to accommodate for typical differences in tissue staining intensities, caused by variations in slide preparation. Unlike Ciompi et al. [20], here we used patches of $256 \times 256$ pixels for classification, which experimentally showed to improve performance and produce a smoother segmentation map (data not shown). Performance of the system was assessed by segmenting all WSI in the dataset in a five-fold cross validation fashion (at WSI level) and evaluating accuracy in all annotated regions.

To enable comparison, the CNN-based TSR-auto was computed in the same circular region (with $1.8 \mathrm{~mm}$ diameter) that was selected by the observers at the consensus meeting, where TSR-visual was assessed. The corresponding image data were extracted from each WSI as circles with a diameter of $\sim 4000$ pixels and processed further by the CNN described above (Fig. 1). Segmentation of a WSI into nine different tissue classes enabled in- and exclusion of specific tissue types comparable to the visual assessment procedure. The used definition of TSRauto is similar to TSR-visual, expressing the area consisting of stroma as a percentage of the area occupied by both tumor and stroma.

\subsection{Statistical analyses}

In this study, TSR-visual and TSR-auto were compared as prognostic factors in rectal cancer. Statistical analyses were performed using IBM SPSS software v24.0 (Armonk, NY, USA). The intraclass correlation coefficient (ICC) was used to determine the correlation between TSR assessed by two observers and by the automated method. To investigate a possible relationship between clinicopathological variables and the numerical values of TSR-visual and TSR-auto, MannWhitney $U$ and Kruskal-Wallis tests were performed for two- and multi-class variables, respectively. For further statistical analysis, TSR-visual and TSR-auto were dichotomized, subdividing patients into two groups: 'stroma-low' and 'stroma-high'. Dichotomization of TSR-visual was performed based on a cut-off value previously established [10] on 63 colon cancer cases: stroma-high $=$ TSR-visual $>50 \%$ and stroma-low $=$ TSR-visual $\leq 50 \%$. In this study, we analyzed results for two different cut-off values for TSR-auto since the optimal cut-off value for the automated approach is not yet established. One method of dichotomization used the ' $50 \%$ stroma cut-off', similar to TSR-visual, referred to as TSRauto(50\%), and the other dichotomization method was based on the median value for all measured TSR-auto values, referred to as TSR-auto(median), yielding equal numbers of patients in stroma-low and stroma-high groups.

Inter-observer agreements were calculated using Cohen's Kappa (к) on the dichotomized TSR values. Kaplan-Meier survival analyses were performed and log-rank statistics were used to test differences in both disease-specific survival (DSS) and disease-free survival (DFS) distributions. DSS was defined as the time between the date of surgery and the date of death attributable to rectal adenocarcinoma. For DFS, the date of the first event of cancer recurrence was used, which could be loco-regional or a distant metastasis. In case no event occurred, the time period until the last date of follow-up was used in the survival analyses. Finally, both uni- and multivariate analyses were performed for TSR-visual and TSR-auto using the Cox proportional hazards model. Probability values $<0.05$ (2-sided) were considered statistically significant.

\section{Results}

\subsection{Clinicopathological data}

Of 154 cases projected for inclusion in this study, twelve cases with mucinous carcinoma were excluded as these 

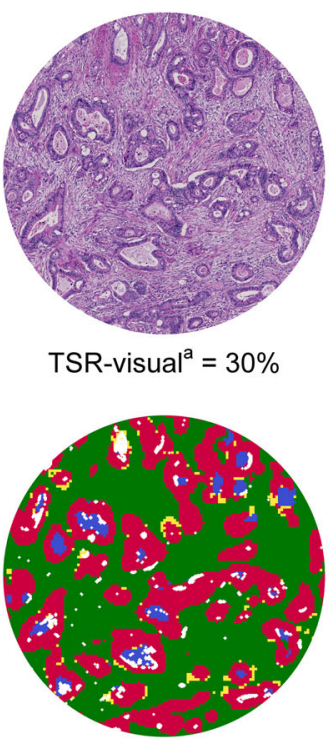

TSR-auto $=58 \%$

$\square=$ Background

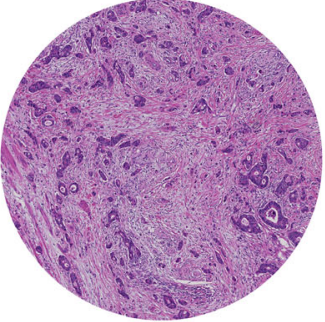

TSR-visual $^{\mathrm{a}}=60 \%$

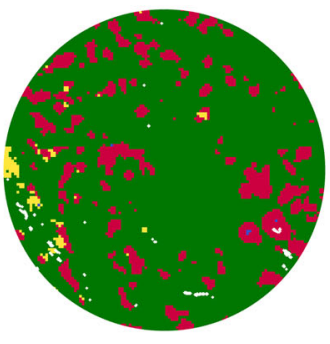

TSR-auto $=84 \%$

= Tumor

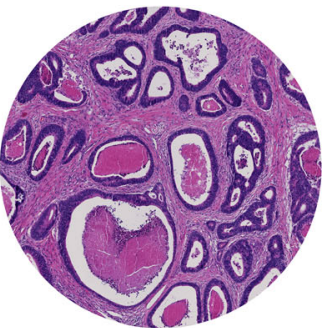

TSR-visual $^{a}=40 \%$

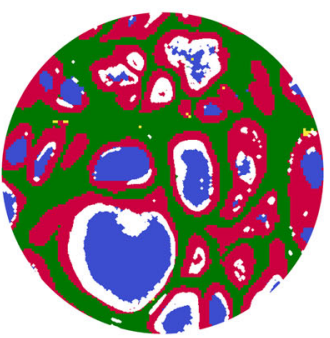

TSR-auto $=50 \%$

$=$ Necrosis

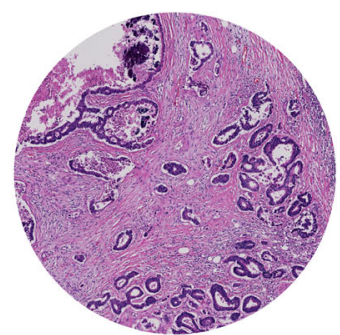

TSR-visual $^{a}=60 \%$

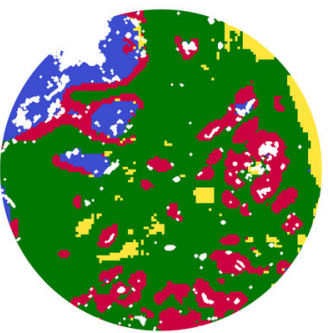

TSR-auto $=78 \%$

$=$ Other tissue ${ }^{b}$

Fig. 1 Top row: Stroma hot-spot circles, $1.8 \mathrm{~mm}$ across, selected by the observers for the assessment of TSR-visual ${ }^{\mathrm{a}}$ and extracted with a diameter of $\sim 4000$ pixels for processing by the CNN. Bottom row: The same

regions with tissues segmented by the $\mathrm{CNN}$ for the calculation of TSRauto. ${ }^{\mathrm{a}}$ Observer consensus; ${ }^{\mathrm{b}}$ Other tissue includes classes: muscle, healthy epithelium, fatty tissue, lymphocytes, mucus and erythrocytes

tumors exhibit largely different TSR values. Twelve other cases were excluded because, at the time of writing, the required slides or data were unavailable. One case was excluded because the corresponding tissue slide did not contain invasive carcinoma.

The median follow-up time for the remaining 129 patients used in the present study was 5.6 years (interquartile range 2.3-8.3). The median age of the patients at the time of surgery was 67 years (interquartile range 59-74). Further clinicopathological data can be found in Table 1. There was no significant correlation between the clinicopathological variables and assessed values of TSR-visual or TSR-auto $(p>0.05)$.

\subsection{Performance of the deep learning system}

Measures of sensitivity and specificity per tissue type as well as overall accuracy were assessed for the automatic method by pixel-wise comparison of predicted labels with ground truth labels in manually annotated regions. We found that the overall accuracy was $94.6 \%$, which shows improvement on what was reported by Ciompi et al. [20]. Values of per-class sensitivity and specificity are reported in Table 2 .

Examples of tissue segmentation by the CNN in four circular regions selected by the observers are shown in Fig. 1. In line with the high classification accuracy, good segmentation of tumor, stroma and other tissues types was observed. Further qualitative inspection of the circular regions revealed some minor segmentation errors. Directly at the stroma-tumor interface, a very thin band of stroma pixels is often misclassified as tumor. Likewise, however, small groups of tumor cells (e.g. tumor buds, or thin tumor structures) were sometimes misclassified as stroma.

\subsection{Inter-observer and computer-observer agreement}

The ICC between the two observers for the assessment of TSR was 0.736 (95\% confidence interval (95\% CI) $0.646-0.806$ ). The co-occurrence of TSR scores assessed by the two observers is depicted in Fig. 2. The ICC's between TSR-auto and TSR-visual were 0.475 (95\% CI $0.330-0.598$ ) and 0.411 (95\% CI $0.257-0.545$ ) for observers 1 and 2 , respectively.

A moderate agreement between the two observers $(\kappa=$ 0.578) was found after dichotomizing TSR-visual on basis of the 50\% cut-off as described in section 2.5. Using the identical cut-off for TSR-auto, we observed only a fair agreement between TSR-visual and TSR-auto $(\kappa=0.239)$. Agreement improved considerably ( $\kappa=0.521$ ) when the median was used as cut-off for TSR-auto, resulting in: stroma-low $=$ TSR-auto $\leq 65.47 \%$ and stroma-high $=$ TSR-auto $>65.47 \%$. Patients assigned to stroma-low or stroma-high groups by the observers and the automatic method are detailed in Tables 3, 4 and 5 .

\subsection{Survival analyses}

Survival analysis generally showed a worse outcome for stroma-high patients compared to stroma-low patients 
Table 1 Clinicopathological data for 129 rectal cancer patients in relation to TSR-visual ${ }^{\mathrm{a}}$ and TSR-auto

\begin{tabular}{|c|c|c|c|c|c|c|c|}
\hline & \multirow{2}{*}{$\begin{array}{l}\text { Total } \\
n(\%)\end{array}$} & \multicolumn{2}{|l|}{ TSR-visual $^{\mathrm{a}}$} & \multicolumn{2}{|c|}{ TSR-auto(50\%) } & \multicolumn{2}{|c|}{ TSR-auto(median) } \\
\hline & & $\begin{array}{l}\text { Stroma-low } \\
n(\%)\end{array}$ & $\begin{array}{l}\text { Stroma-high } \\
n(\%)\end{array}$ & $\begin{array}{l}\text { Stroma-low } \\
n(\%)\end{array}$ & $\begin{array}{l}\text { Stroma-high } \\
n(\%)\end{array}$ & $\begin{array}{l}\text { Stroma-low } \\
n(\%)\end{array}$ & $\begin{array}{l}\text { Stroma-high } \\
n(\%)\end{array}$ \\
\hline \multicolumn{8}{|l|}{ Gender } \\
\hline Female & $43(34)$ & $30(34)$ & $13(31)$ & $11(35)$ & $32(33)$ & $22(34)$ & $21(32)$ \\
\hline Male & $86(67)$ & $57(66)$ & $29(69)$ & $20(65)$ & $66(67)$ & $42(66)$ & $44(68)$ \\
\hline \multicolumn{8}{|l|}{ T-status } \\
\hline pT1 & $4(3)$ & $4(5)$ & $0(0)$ & $2(6)$ & $2(2)$ & $3(5)$ & $1(2)$ \\
\hline pT2 & $40(31)$ & $29(33)$ & $11(26)$ & $5(16)$ & $35(36)$ & $18(28)$ & $22(34)$ \\
\hline pT3 & $79(61)$ & $51(59)$ & $28(67)$ & $24(77)$ & $55(56)$ & $42(66)$ & $37(57)$ \\
\hline pT4 & $6(5)$ & $3(3)$ & $3(7)$ & $0(0)$ & $6(6)$ & $1(2)$ & $5(8)$ \\
\hline \multicolumn{8}{|l|}{ N-status } \\
\hline pN0 & $78(60)$ & $54(62)$ & $24(57)$ & $22(71)$ & $56(57)$ & $43(67)$ & $35(54)$ \\
\hline $\mathrm{pN} 1$ & $33(26)$ & $23(26)$ & $10(24)$ & $6(19)$ & $27(28)$ & $13(20)$ & $20(31)$ \\
\hline $\mathrm{pN} 2$ & $18(14)$ & $10(11)$ & $8(19)$ & $3(10)$ & $15(15)$ & $8(13)$ & $10(15)$ \\
\hline \multicolumn{8}{|l|}{ Stage } \\
\hline $\mathrm{I}$ & $33(26)$ & $26(30)$ & $7(17)$ & $6(19)$ & $27(28)$ & $18(28)$ & $15(23)$ \\
\hline II & $45(35)$ & $28(32)$ & $17(40)$ & $16(52)$ & $29(30)$ & $25(39)$ & $20(31)$ \\
\hline III & $51(40)$ & $33(38)$ & $18(43)$ & $9(29)$ & $42(43)$ & $21(33)$ & $30(46)$ \\
\hline \multicolumn{8}{|l|}{ Tumor grade } \\
\hline Well & $3(2)$ & $2(2)$ & $1(2)$ & $1(3)$ & $2(2)$ & $1(2)$ & $2(3)$ \\
\hline Moderate & $112(87)$ & $73(84)$ & $39(93)$ & $28(90)$ & $84(86)$ & $55(86)$ & $57(88)$ \\
\hline Poor & $14(11)$ & $12(14)$ & $2(5)$ & $2(6)$ & $12(12)$ & $8(13)$ & $6(9)$ \\
\hline \multicolumn{8}{|l|}{ Surgery type } \\
\hline APR & $62(48)$ & $39(45)$ & $23(55)$ & $10(32)$ & $52(53)$ & $26(41)$ & $36(55)$ \\
\hline LAR & $49(38)$ & $37(43)$ & $12(29)$ & $17(55)$ & $32(33)$ & $29(45)$ & $20(31)$ \\
\hline Hartmann & $18(14)$ & $11(13)$ & $7(17)$ & $4(13)$ & $14(14)$ & $9(14)$ & $9(14)$ \\
\hline \multicolumn{8}{|l|}{ Adjuvant treatment } \\
\hline None & $86(67)$ & $59(68)$ & $27(64)$ & $24(77)$ & $62(63)$ & $45(70)$ & $41(63)$ \\
\hline Radiotherapy & $43(33)$ & $28(32)$ & $15(36)$ & $7(23)$ & $36(37)$ & $19(30)$ & $24(37)$ \\
\hline Chemoradioth. $^{\mathrm{b}}$ & $5(4)$ & $3(3)$ & $2(5)$ & $1(3)$ & $4(4)$ & $2(3)$ & $3(5)$ \\
\hline
\end{tabular}

Mann-Whitney U and Kruskal-Wallis tests showed no significant correlation $(p>0.05)$ between the listed variables and TSR-visual or TSR-auto LAR: Low anterior resection; APR: Abdominoperineal resection; pT: Pathological tumor status; pN: Pathological nodal status

${ }^{a}$ Observer consensus

${ }^{\mathrm{b}}$ Chemoradiotherapy

(Fig. 3), independent of the method of TSR assessment used (visual versus automated). For TSR-visual, the 5-year survival rates for stroma-low versus stroma-high cases were $71.0 \%$ versus $58.8 \%$ for DSS and $65.6 \%$ versus $49.1 \%$ for DFS. For TSR-auto(50\%), the 5-year survival rates for stroma-low versus stroma-high cases were $86.6 \%$ versus $60.7 \%$ for DSS and $76.8 \%$ versus $54.9 \%$ for DFS. For TSR-auto(median), the 5 -year survival rates for stroma-low versus stroma-high cases, were $76.1 \%$ versus $58.4 \%$ for DSS and $70.0 \%$ versus $50.7 \%$ for DFS.
Table 2 Quantitative performance of the $\mathrm{CNN}$ at pixel classification per tissue class

\begin{tabular}{llllllllll}
\hline & Tumor & Stroma & Necrosis & Muscle & $\begin{array}{l}\text { Healthy } \\
\text { epi. }\end{array}$ & Fat & Mucus & $\begin{array}{l}\text { Lympho- } \\
\text { cytes }\end{array}$ & Blood \\
\hline Sensitivity & $91.1 \%$ & $91.7 \%$ & $90.8 \%$ & $95.5 \%$ & $94.1 \%$ & $98.1 \%$ & $96.4 \%$ & $98.4 \%$ & $97.9 \%$ \\
Specificity & $99.4 \%$ & $97.7 \%$ & $99.6 \%$ & $99.6 \%$ & $99.5 \%$ & $99.9 \%$ & $98.7 \%$ & $99.6 \%$ & $99.9 \%$ \\
\hline
\end{tabular}

CNN: Convolutional Neural Network; Healthy epi.: Healthy epithelium 


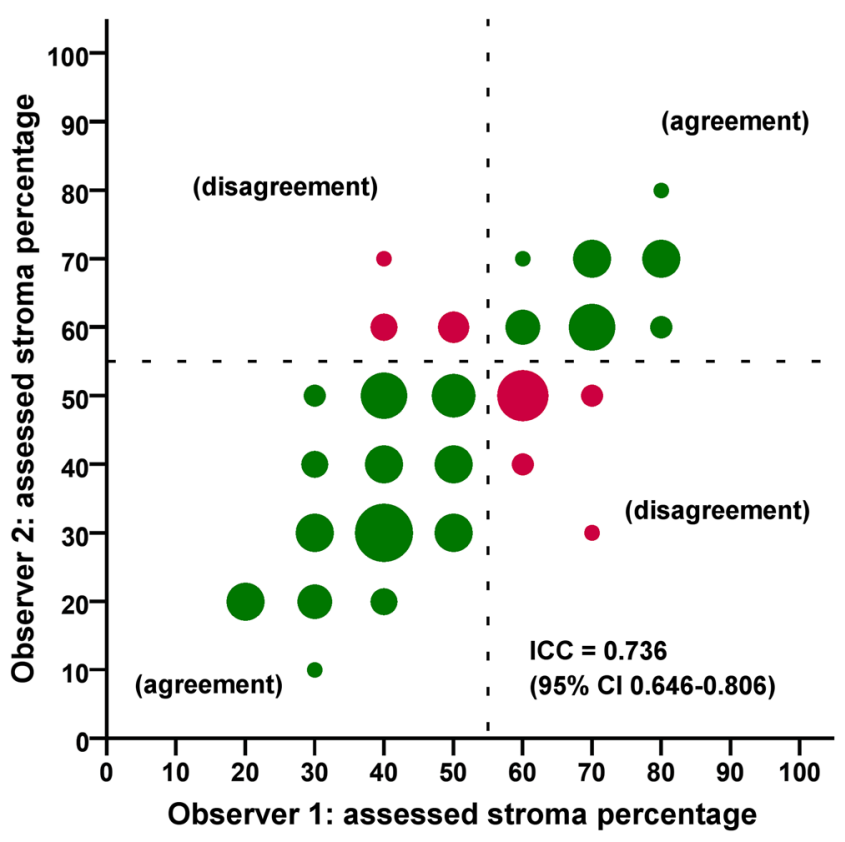

Fig. 2 Scatter plot of assessed stroma percentages in 129 patients for Observer 1 and Observer 2. The co-occurrence of assessed percentages is indicated by circles with areas proportional to the amount of patients scored with the corresponding TSR value. The dashed lines represent the boundary between stroma-low and stroma-high cases according to the cut-off value determined in Mesker et al. [10]. Green circles indicate cases where the observers agreed (105 in total) and red circles indicate disagreement (24 in total)

For TSR-visual, a significantly lower DSS was seen in the stroma-high group compared to the stroma-low group ( $p=$ $0.042)$, but not for DFS $(p=0.182)$. Similarly, for TSR-auto $(50 \%)$ this difference was significant for DSS $(p=0.018)$, but not for DFS ( $p=0.066)$. For TSR-auto(median), both DSS and DFS were found to be significantly lower in the stromahigh group compared to the stroma-low group $(p=0.007$ and $p=0.021$, respectively). After stratification for TNM stage, stroma-high was also found to be associated with worse survival in stage II rectal cancer patients $(n=45)$, but this result was only significant for TSR-auto(median) (DSS $p=0.003$ and DFS $p=0.015$ ).

Hazard ratios (HR) and 95\% CIs were determined for both DSS and DFS (Tables 6 and 7). In univariate analysis, all

Table 3 Cross-tabulation of Observer 1 versus Observer 2 after dichotomisation

\begin{tabular}{lllll}
\hline \multirow{2}{*}{$\kappa=0.578$} & \multicolumn{2}{l}{ Observer 2 } & \\
\cline { 3 - 5 } & & $\begin{array}{l}\text { Stroma- } \\
\text { low }\end{array}$ & $\begin{array}{l}\text { Stroma- } \\
\text { high }\end{array}$ & Total \\
\hline Observer 1 & Stroma-low & 75 & 8 & 83 \\
& Stroma-high & 16 & 30 & 46 \\
& Total & 91 & 38 & 129 \\
\hline
\end{tabular}

Table 4 Cross-tabulation of TSR-visual (consensus) versus TSR-auto $(50 \%)$ after dichotomisation

\begin{tabular}{lllll}
\hline & $\kappa=0.239$ & \multicolumn{2}{l}{ TSR-auto(50\%) } \\
\cline { 3 - 5 } \cline { 3 - 5 } & & $\begin{array}{l}\text { Stroma- } \\
\text { low }\end{array}$ & $\begin{array}{l}\text { Stroma- } \\
\text { high }\end{array}$ & Total \\
\hline TSR-visual (consensus) & Stroma-low & 30 & 57 & 87 \\
& Stroma-high & 1 & 41 & 42 \\
& Total & 31 & 98 & 129 \\
\hline
\end{tabular}

methods for TSR assessment were found to be prognostic for DSS: TSR-visual HR $=1.83$ (95\% CI 1.01-3.30); TSRauto $(50 \%)$ HR $=2.71$ (95\% CI 1.14-6.40); and TSRauto(median) $\mathrm{HR}=2.31$ (95\% CI 1.24-4.30). For DFS, only TSR-auto(median) was found to be prognostic with $\mathrm{HR}=1.96$ (95\% CI 1.10-3.51). After stratification for TNM stage, only TSR-auto(median) was found to be prognostic for stage II rectal cancer patients, both for DSS (univariate HR $=4.13$ (95\% CI 1.53-11.16)) and DFS (univariate $\mathrm{HR}=3.05$ (95\% CI 1.19-7.81)).

In multivariate analysis, automated TSR assessment was found to be prognostic independent of age, gender, pT-stage, lymph node status, tumor grade, and whether adjuvant therapy was given, both for DSS: TSR-auto(50\%) $\mathrm{HR}=3.11$ (95\% CI 1.26-7.70) and TSR-auto(median) $\mathrm{HR}=2.48$ (95\% CI 1.29-4.78), and for DFS: TSR-auto $(50 \%)(\mathrm{HR}=2.39(95 \% \mathrm{CI} \quad 1.07-5.38))$ and TSRauto(median) $(\mathrm{HR}=2.05$ (95\% CI 1.11-3.78)). TSR-visual was not found to serve as an independent prognostic factor.

\section{Discussion}

For different cancer types, TSR has been shown to yield prognostic information. Visual assessment of TSR requires training, and may be difficult for cases close to the decision threshold of $50 \%$. The present study shows that specifically for rectal adenocarcinoma the observer agreement is only moderate. Recent advances in slide scanning technology and machine learning have opened up new possibilities for computerized assessment of TSR. To the best of our knowledge, the

Table 5 Cross-tabulation of TSR-visual (consensus) versus TSRauto(median) after dichotomisation

\begin{tabular}{lllll}
\hline & $\kappa=0.521$ & \multicolumn{2}{l}{ TSR-auto(median) } \\
\cline { 3 - 5 } \cline { 3 - 5 } & & $\begin{array}{l}\text { Stroma- } \\
\text { low }\end{array}$ & $\begin{array}{l}\text { Stroma- } \\
\text { high }\end{array}$ & Total \\
\hline TSR-visual (consensus) & Stroma-low & 60 & 27 & 87 \\
& Stroma-high & 4 & 38 & 42 \\
& Total & 64 & 65 & 129 \\
\hline
\end{tabular}



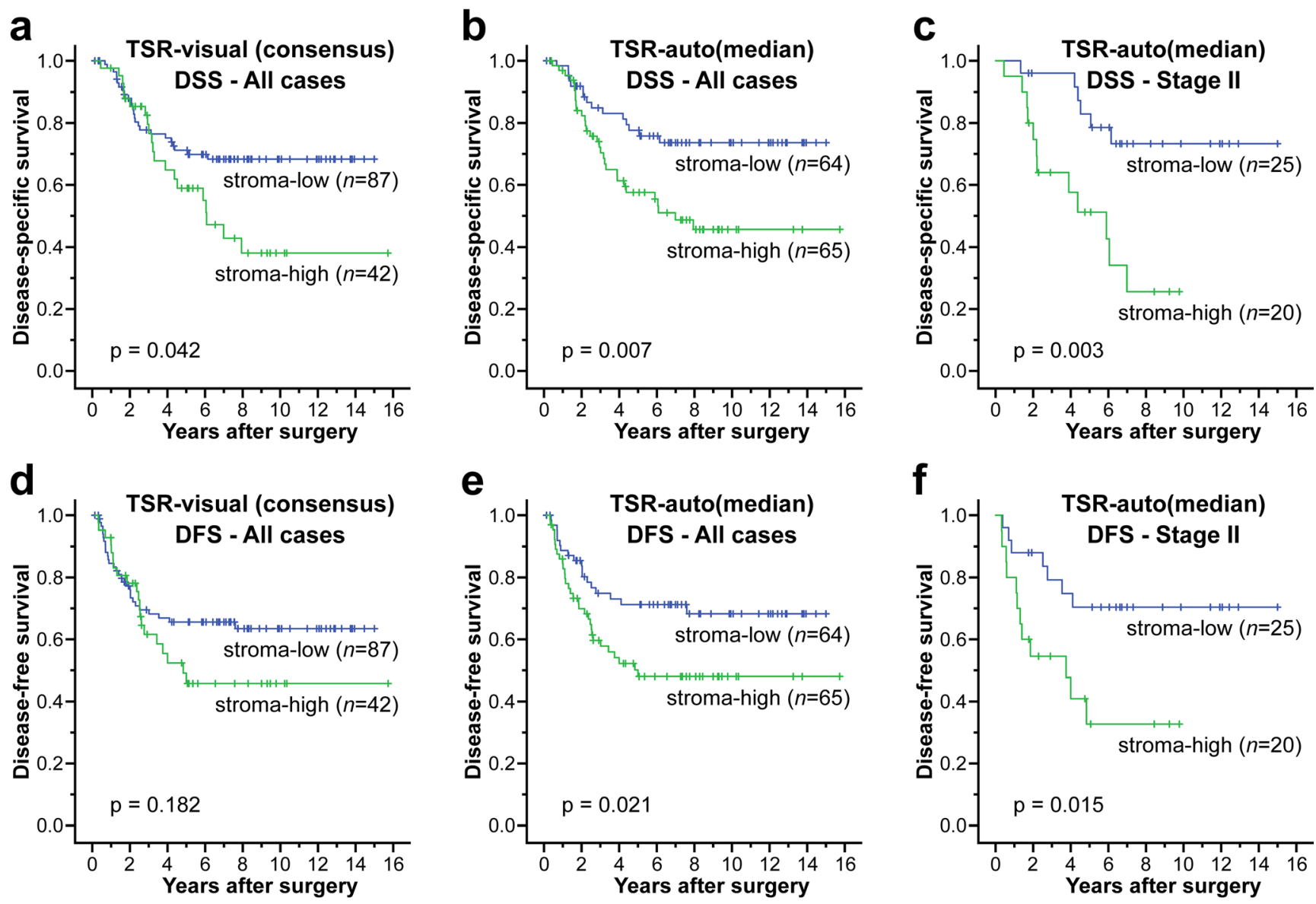

Fig. 3 Kaplan-Meier curves for disease-specific survival (top row) and disease-free survival (bottom row) of stroma-low versus stroma-high patients. Results based on all patients $(n=129)$ are shown for TSR-visual (a,

present study shows for the first time that TSR can reliably be assessed by an automatic deep learning algorithm. The agreement of the automated system (using median cut-off) with the observer consensus $(\mathrm{kappa}=0.521)$ was comparable to the inter-observer agreement $(\mathrm{kappa}=0.578)$. The TSR assessed in this manner appeared to be a strong independent prognostic factor both for DSS and DFS in rectal adenocarcinoma. The prognostic value of the automated TSR was comparable to that assessed in consensus by two experienced observers for DSS in univariate analysis, but not in multivariate analysis. For DFS, only the automatically assessed TSR was significantly associated with outcome, both in univariate and multivariate analysis.

Interestingly, automated TSR (using the median as cut-off) showed prognostic value for TNM stage II patients. Clinically, this is a subgroup of patients for which post-operative treatment is still under debate and more research is needed $[22,23]$. TSR can potentially help to direct this discussion and add information for a more personalized treatment of this patient category.

In a recent study, Scheer et al. [8] analyzed TSR on the same cohort of patients as used in the present study. However, rather d) and TSR-auto(median) (b, e). Results for patients with stage II rectal cancer $(n=45)$ are shown for TSR-auto(median) only $(\mathbf{c}, \mathbf{f})$. Log-rank test $p$-values are shown in the graphs

than a hot-spot measure, the authors applied a scoring procedure in which an average TSR was assessed based on the entire tumor area in a slide. Also, they defined TSR as the carcinoma percentage $(\mathrm{CP})$ and the estimated percentages were grouped using three categories (low-CP, intermediate-CP and high-CP). In univariate survival analysis, $\mathrm{CP}$ was found to be prognostic for DSS and DFS. With CP-high as baseline and after correction for age, grading, pathological T-stage, and adjuvant treatment, CPintermediate was found to be correlated with worse DSS and DFS, however, this result was obtained only in the subset of lymph node metastasis negative cases $(n=94)$. In the present study, the prognostic value of TSR remained intact for the entire cohort of patients after correction for clinicopathological variables, including lymph node status. The most probable cause for this difference is the TSR scoring method. In the present study we decided to follow a more widely accepted scoring system, which appears to outperform methods where the overall tumor area is scored by averaging.

The results of our observer study indicate that TSR obtained by visual estimation serves as a prognostic factor of DSS (although not reaching statistical significance when correcting for 
Table 6 Uni- and multivariate Cox regression analysis for disease-specific survival

\begin{tabular}{|c|c|c|c|c|c|c|c|c|}
\hline & \multirow{2}{*}{\multicolumn{2}{|c|}{ Univariate }} & \multicolumn{6}{|c|}{ Multivariate } \\
\hline & & & \multicolumn{2}{|c|}{ TSR-visual $^{\mathrm{a}}$} & \multicolumn{2}{|c|}{ TSR-auto(50\%) } & \multicolumn{2}{|c|}{ TSR-auto(median) } \\
\hline & HR & $95 \% \mathrm{CI}$ & HR & $95 \% \mathrm{CI}$ & HR & $95 \% \mathrm{CI}$ & HR & $95 \% \mathrm{CI}$ \\
\hline $\mathrm{Age}^{\mathrm{b}}$ & 1.01 & $0.98-1.04$ & 1.01 & $0.98-1.04$ & 1.00 & $0.98-1.03$ & 1.01 & $0.98-1.04$ \\
\hline \multicolumn{9}{|l|}{ Gender } \\
\hline Female & 1.00 & & 1.00 & & 1.00 & & 1.00 & \\
\hline Male & 1.16 & $0.61-2.17$ & 1.64 & $0.83-3.24$ & 1.67 & $0.83-3.34$ & 1.62 & $0.83-3.18$ \\
\hline \multicolumn{9}{|l|}{ T-status ${ }^{c}$} \\
\hline pT1-pT2 & 1.00 & & 1.00 & & 1.00 & & 1.00 & \\
\hline pT3-pT4 & 4.52 & $1.91-10.72$ & 3.75 & $1.56-9.03$ & 4.80 & $1.96-11.75$ & 4.48 & $1.84-10.91$ \\
\hline \multicolumn{9}{|l|}{ LN metastases ${ }^{\mathrm{d}}$} \\
\hline No & 1.00 & & 1.00 & & 1.00 & & 1.00 & \\
\hline Yes & 2.93 & $1.35-6.32$ & 1.64 & $0.53-5.08$ & 1.43 & $0.45-4.57$ & 1.26 & $0.42-3.76$ \\
\hline \multicolumn{9}{|l|}{ Tumor grade $\mathrm{e}^{\mathrm{e}}$} \\
\hline Well-Moderate & 1.00 & & 1.00 & & 1.00 & & 1.00 & \\
\hline Poor & 2.86 & $1.32-6.20$ & 2.87 & $1.24-6.69$ & 2.29 & $0.96-5.47$ & 2.63 & $1.14-6.08$ \\
\hline \multicolumn{9}{|l|}{ Adjuvant therapy } \\
\hline No & 1.00 & & 1.00 & & 1.00 & & 1.00 & \\
\hline Yes & 2.31 & $1.28-4.15$ & 1.28 & $0.39-4.17$ & 1.25 & $0.37-4.22$ & 1.42 & $0.44-4.54$ \\
\hline \multicolumn{9}{|l|}{ TSR-visual $^{\mathrm{a}}$} \\
\hline Stroma-low & 1.00 & & 1.00 & & & & & \\
\hline Stroma-high & 1.83 & $1.01-3.30$ & 1.76 & $0.93-3.34$ & & & & \\
\hline \multicolumn{9}{|l|}{ TSR-auto(50\%) } \\
\hline Stroma-low & 1.00 & & & & 1.00 & & & \\
\hline Stroma-high & 2.71 & $1.14-6.40$ & & & 3.11 & $1.26-7.70$ & & \\
\hline \multicolumn{9}{|l|}{ TSR-auto(median) } \\
\hline Stroma-low & 1.00 & & & & & & 1.00 & \\
\hline Stroma-high & 2.31 & $1.24-4.30$ & & & & & 2.48 & $1.29-4.78$ \\
\hline
\end{tabular}

${ }^{\mathrm{a}}$ Observer consensus

${ }^{\mathrm{b}}$ Age was used as a continuous variable

${ }^{\mathrm{c}}$ Due to low numbers, pT1 $(n=4)$ and pT2 cases were grouped together as well as pT3 and pT4 $(n=6)$ cases

${ }^{\mathrm{d}}$ Lymph node metastases

${ }^{\mathrm{e}}$ Due to low numbers, cases with well $(n=3)$ and moderately differentiated tumors were grouped together

Significant results $(p>0.05)$ are indicated in bold

other clinicopathological features), but not of DFS. Furthermore, only a moderate agreement was found between observers. These results are in contrast with previous studies [9, 10, 13] on TSR assessment on colon cancer. This discrepancy may be explained by the fact that compared to colon, the rectum bowel wall has a thicker muscle layer and in, some cases, it may be difficult to distinguish between stromal tissue and smooth muscle cells, especially with darker H\&E-stained slides. Muscle tissue, which should be excluded from scoring, may therefore be interpreted as stromal tissue by one observer and not by the other. Furthermore, as shown in Fig. 2, most discrepancies (15/24 cases) are found around the cut-off point of $50 \%$. Especially for these cases, computer-aided TSR assessment may be very useful.

For the automated method two different stroma cut-off values have been investigated in this study: the value used for the visual estimation $(50 \%)$, and the median of measured TSR-auto values. We found comparable results for the two cut-offs, with a slightly higher hazard ratio for the $50 \%$ cutoff at the cost of a wider $95 \%$ confidence interval. However, since in general automated assessment of TSR yields higher stroma percentages than visual assessment, the use of a $50 \%$ cut-off for TSR-auto corresponded much less to TSR-visual 
Table 7 Uni- and multivariate Cox regression analysis for disease-free survival

\begin{tabular}{|c|c|c|c|c|c|c|c|c|}
\hline & \multirow{2}{*}{\multicolumn{2}{|c|}{ Univariate }} & \multicolumn{6}{|c|}{ Multivariate } \\
\hline & & & \multicolumn{2}{|c|}{ TSR-visual $^{\mathrm{a}}$} & \multicolumn{2}{|c|}{ TSR-auto(50\%) } & \multicolumn{2}{|c|}{ TSR-auto(median) } \\
\hline & HR & $95 \% \mathrm{CI}$ & HR & $95 \% \mathrm{CI}$ & HR & $95 \% \mathrm{CI}$ & HR & $95 \% \mathrm{CI}$ \\
\hline $\mathrm{Age}^{\mathrm{b}}$ & 1.00 & $0.97-1.02$ & 1.00 & $0.97-1.02$ & 0.99 & $0.96-1.02$ & 0.99 & $0.97-1.02$ \\
\hline \multicolumn{9}{|l|}{ Gender } \\
\hline Female & 1.00 & & 1.00 & & 1.00 & & 1.00 & \\
\hline Male & 1.35 & $0.73-2.52$ & 1.87 & $0.96-3.63$ & 1.80 & $0.92-3.52$ & 1.81 & $0.94-3.49$ \\
\hline \multicolumn{9}{|l|}{ T-status ${ }^{c}$} \\
\hline pT1-pT2 & 1.00 & & 1.00 & & 1.00 & & 1.00 & \\
\hline pT3-pT4 & 4.09 & $1.83-9.12$ & 3.52 & $1.55-8.01$ & 4.33 & $1.87-10.04$ & 4.11 & $1.78-9.48$ \\
\hline \multicolumn{9}{|l|}{ LN metastases ${ }^{\mathrm{d}}$} \\
\hline No & 1.00 & & 1.00 & & 1.00 & & 1.00 & \\
\hline Yes & 2.43 & $1.38-4.28$ & 1.89 & $0.68-5.25$ & 1.78 & $0.63-5.00$ & 1.55 & $0.58-4.19$ \\
\hline \multicolumn{9}{|l|}{ Tumor grade $^{\mathrm{e}}$} \\
\hline Well-Moderate & 1.00 & & 1.00 & & 1.00 & & 1.00 & \\
\hline Poor & 2.24 & $1.05-4.80$ & 2.02 & $0.88-4.62$ & 1.65 & $0.70-3.87$ & 1.78 & $0.78-4.06$ \\
\hline \multicolumn{9}{|l|}{ Adjuvant therapy } \\
\hline No & 1.00 & & 1.00 & & 1.00 & & 1.00 & \\
\hline Yes & 2.22 & $1.26-3.89$ & 1.07 & $0.37-3.10$ & 0.98 & $0.33-2.90$ & 1.11 & $0.39-3.15$ \\
\hline \multicolumn{9}{|l|}{ TSR-visual $^{\text {a }}$} \\
\hline Stroma-low & 1.00 & & 1.00 & & & & & \\
\hline Stroma-high & 1.47 & $0.83-2.61$ & 1.42 & $0.77-2.61$ & & & & \\
\hline \multicolumn{9}{|l|}{ TSR-auto(50\%) } \\
\hline Stroma-low & 1.00 & & & & 1.00 & & & \\
\hline Stroma-high & 2.01 & $0.94-4.29$ & & & 2.39 & $1.07-5.38$ & & \\
\hline \multicolumn{9}{|l|}{ TSR-auto(median) } \\
\hline Stroma-low & 1.00 & & & & & & 1.00 & \\
\hline Stroma-high & 1.96 & $1.10-3.51$ & & & & & 2.05 & $1.11-3.78$ \\
\hline
\end{tabular}

${ }^{\mathrm{a}}$ Observer consensus

${ }^{\mathrm{b}}$ Age was used as a continuous variable

${ }^{\mathrm{c}}$ Due to low numbers, pT1 $(n=4)$ and pT2 cases were grouped together as well as pT3 and pT4 $(n=6)$ cases

${ }^{\mathrm{d}}$ Lymph node metastases

${ }^{\mathrm{e}}$ Due to low numbers, cases with well $(n=3)$ and moderately differentiated tumors were grouped together

Significant results $(p>0.05)$ are indicated in bold

compared to the use of the median cut-off (as is reflected in the kappa values). The optimal cut-off value for TSR-auto should be further investigated and validated in an independent cohort.

It is worth noting that one of the patient inclusion criteria for the cohort that was used in this study was the absence of neoadjuvant treatment. The reason for this design choice, originally made by Scheer et al. [8], was that both chemotherapy and radiotherapy modifies the tissue architecture and, as such, may hamper the assessment of TSR or its prognostic value. The proposed method can, therefore, aid clinicians in selecting the right treatment options for rectal cancer patients who did not receive preoperative (chemo)radiotherapy. Furthermore, given the fact that the colon and the rectum are parts of the same continuous organ and have a similar histological appearance, the presented deep learning algorithm has the potential to be successfully applied to the analysis of colon cancer as well.

The deep learning-based approach proposed in this work needs the position of a user-provided stroma hot-spot as input in order to assess TSR. After this manual input is provided, the proposed method can process the hot-spot area in the whole-slide image automatically. As such, human input is still required, making the method only semi-automatic. It is worth noting that in Ciompi et al. [20] a computer model similar to the one used in 
this work has shown a high performance at segmenting several tissue types in rectal cancer at the whole-slide image level, i.e., beyond the limited area of the selected hot-spot. As a consequence, this method has the potential to be used to assess TSR both at whole-tumor level and at whole-slide image level. Such an approach would overcome the need for a user-provided stroma hot-spot and, therefore, allow investigating TSR at very large scale via fully-automatic computation. Future work will be directed towards further automation of TSR assessment and validation in a large independent cohort.

Although, to the best of our knowledge, TSR assessment (visual or automated) has not yet been implemented in routine pathology diagnostics, it was recently reported [24] that the TNM Evaluation Committee (UICC) and the College of American Pathologists (CAP) have discussed TSR and acknowledged its potential for integration with the TNM staging system. To achieve this for colon cancers, we are currently investigating the reproducibility of (visual) TSR assessment in a large European multicenter study [25]. The results of the present study suggest that automated TSR can potentially be of significant aid to pathologists in routine diagnostics. However, validation of the proposed technology on a larger and independent data set is essential and, therefore, among our future research goals. The objectiveness of a deep learning-based method, which allows obtaining accurate and reproducible quantification of TSR, has the potential to pave the way to implementation of TSR in clinical practice.

Acknowledgements The authors would like to thank Gabriel Silva de Souza for his help in making manual annotations. We would also like to thank NVIDIA for the donation of a GeForce TITAN Xp card, which was used to train the convolutional networks used in this work.

Author's contributions JvdL, FC, OG, AB and IN designed the study. JK provided the clinical data. $\mathrm{OG}$ and $\mathrm{AB}$ made and checked the manual annotations. FC, BEB, GL developed the deep learning algorithm. GvP and WM visually assessed TSR. OG and JvdL analyzed the data. OG, JvdL and FC interpreted the results. OG, FC and JvdL wrote the manuscript. All authors critically reviewed the manuscript and approved the final version.

Funding $\mathrm{FC}$ and OG were supported by a grant from the Dutch Cancer Society (KWF) / Alpe d'HuZes fund (KUN 2014-7032).

\section{Compliance with ethical standards}

Conflict of interest JvdL is a member of the scientific advisory boards of Philips, The Netherlands and ContextVision, Sweden. JvdL receives research funding from Sectra, Sweden and receives project remuneration from Philips, The Netherlands. The other authors declare that they have no conflict of interest.

Ethical approval All clinical data and microscopy slides were handled in an anonymous (coded) fashion. In accordance with Dutch law and national ethical guidelines ("Code for Proper Secondary Use of Human Tissue", Dutch Federation of Medical Scientific Societies) and the Helsinki Declaration, no formal patient consent was required for this retrospective study.
Open Access This article is distributed under the terms of the Creative Commons Attribution 4.0 International License (http:// creativecommons.org/licenses/by/4.0/), which permits unrestricted use, distribution, and reproduction in any medium, provided you give appropriate credit to the original author(s) and the source, provide a link to the Creative Commons license, and indicate if changes were made.

Publisher's note Springer Nature remains neutral with regard to jurisdictional claims in published maps and institutional affiliations.

\section{References}

1. L.H. Sobin, I.D. Fleming, TNM classification of malignant tumors. Fifth edition Cancer 80, 1803-1804 (1997)

2. H.J. Schmoll, E. van Cutsem, A. Stein, V. Valentini, B. Glimelius, K. Haustermans, B. Nordlinger, C.J. van de Velde, J. Balmana, J. Regula, I.D. Nagtegaal, R.G. Beets-Tan, D. Arnold, F. Ciardiello, P. Hoff, D. Kerr, C.H. Köhne, R. Labianca, T. Price, W. Scheithauer, A. Sobrero, J. Tabernero, D. Aderka, S. Barroso, G. Bodoky, J.Y. Douillard, H. El ghazaly, J. Gallardo, A. Garin, R. Glynne-jones, K. Jordan, A. Meshcheryakov, D. Papamichail, P. Pfeiffer, I. Souglakos, S. Turhal, A. Cervantes, ESMO consensus guidelines for management of patients with colon and rectal cancer. A personalized approach to clinical decision making. Ann. Oncol. 23, 2479 $2516(2012)$

3. F.J.A. Gujam, J. Edwards, Z.M.A. Mohammed, J.J. Going, D.C. McMillan, The relationship between the tumour stroma percentage, clinicopathological characteristics and outcome in patients with operable ductal breast cancer. Br. J. Cancer 111, 157-165 (2014)

4. A.M. Moorman, R. Vink, H.J. Heijmans, J. Van Der Palen, E.A. Kouwenhoven, The prognostic value of tumour-stroma ratio in triple-negative breast cancer. Eur. J. Surg. Oncol. 38, 307-313 (2012)

5. T. Roeke, M. Sobral-Leite, T.J.A. Dekker, J. Wesseling, V.T.H.B.M. Smit, R.A.E.M. Tollenaar, M.K. Schmidt, W.E. Mesker, The prognostic value of the tumour-stroma ratio in primary operable invasive cancer of the breast: A validation study. Breast Cancer Res. Treat. 166, 435-445 (2017)

6. Z. Wang, H. Liu, R. Zhao, H. Zhang, C. Liu, Y. Song, Tumorstroma ratio is an independent prognostic factor of non-small cell lung cancer. Chin. J. Lung Cancer 16, 191-196 (2013)

7. T. Zhang, J. Xu, H. Shen, W. Dong, Y. Ni, J. Du, Tumor-stroma ratio is an independent predictor for survival in NSCLC. Int. J. Clin. Exp. Pathol. 8, 11348-11355 (2015)

8. R. Scheer, A. Baidoshvili, S. Zoidze, M.A. Elferink, A.E. Berkel, J.M. Klaase, P.J. van Diest, Tumor-stroma ratio as prognostic factor for survival in rectal adenocarcinoma: A retrospective cohort study. World J. Gastrointest. Oncol. 9, 466-474 (2017)

9. A. Huijbers, R.A.E.M. Tollenaar, G.W. van Pelt, E.C.M. Zeestraten, S. Dutton, C.C. McConkey, E. Domingo, V.T.H.B.M. Smit, R. Midgley, B.F. Warren, E.C. Johnstone, D.J. Kerr, W.E. Mesker, The proportion of tumor-stroma as a strong prognosticator for stage II and III colon cancer patients: Validation in the victor trial. Ann. Oncol. 24, 179-185 (2013)

10. W.E. Mesker, J.M.C. Junggeburt, K. Szuhai, P. de Heer, H. Morreau, H.J. Tanke, R.A.E.M. Tollenaar, The carcinoma-stromal ratio of colon carcinoma is an independent factor for survival compared to lymph node status and tumor stage. Cell. Oncol. 29, 387398 (2007)

11. J.H. Park, C.H. Richards, D.C. McMillan, P.G. Horgan, C.S.D. Roxburgh, The relationship between tumour stroma percentage, 
the tumour microenvironment and survival in patients with primary operable colorectal cancer. Ann. Oncol. 25, 644-651 (2014)

12. N.P. West, M. Dattani, P. McShane, G. Hutchins, J. Grabsch, W. Mueller, D. Treanor, P. Quirke, H. Grabsch, The proportion of tumour cells is an independent predictor for survival in colorectal cancer patients. Br. J. Cancer 102, 1519-1523 (2010)

13. G.W. van Pelt, T.F. Hansen, E. Bastiaannet, S. Kjær-Frifeldt, J.H.J.M. van Krieken, R.A.E.M. Tollenaar, F.B. Sørensen, W.E. Mesker, Stroma-high lymph node involvement predicts poor survival more accurately for patients with stage III colon cancer. J. Med. Surg. Pathol. 1, 1-8 (2016)

14. O. G. F. Geessink, A. Baidoshvili, G. Freling, J. M. Klaase, C. H. Slump, and F. van der Heijden, Toward automatic segmentation and quantification of tumor and stroma in whole-slide images of $H \& E$ stained rectal carcinomas. Proc. SPIE medical imaging: Digital pathology. 9420, 0F1-0F7 (2015)

15. A. Wright, D. Magee, P. Quirke, and D. E. Treanor, Towards automatic patient selection for chemotherapy in colorectal cancer trials. Proc. SPIE medical imaging: Digital Pathology. 9041, 0A1-0A8 (2014)

16. F. Bianconi, A. Álvarez-Larrán, A. Fernández, Discrimination between tumour epithelium and stroma via perception-based features. Neurocomputing 154, 119-126 (2015)

17. N. Linder, J. Konsti, R. Turkki, E. Rahtu, M. Lundin, S. Nordling, C. Haglund, T. Ahonen, M. Pietikäinen, J. Lundin, Identification of tumor epithelium and stroma in tissue microarrays using texture analysis. Diagn. Pathol. 7, 22 (2012)

18. B. Ehteshami Bejnordi, M. Veta, P.J. van Diest, B. van Ginneken, N. Karssemeijer, G. Litjens, J.A.W.M. van der Laak, M. Hermsen, Q.F. Manson, M. Balkenhol, O.G.F. Geessink, N. Stathonikos, M.C.R.F. van Dijk, P. Bult, F. Beca, A.H. Beck, D. Wang, A. Khosla, R. Gargeya, H. Irshad, A. Zhong, Q. Dou, Q. Li, H. Chen, H.J. Lin, P.A. Heng, C. Haß, E. Bruni, Q. Wong, U. Halici, M.Ü. Öner, R. Cetin-Atalay, M. Berseth, V. Khvatkov, A. Vylegzhanin, O. Kraus, M. Shaban, N. Rajpoot, R. Awan, K. Sirinukunwattana, T. Qaiser, Y.W. Tsang, D. Tellez, J. Annuscheit, P. Hufnagl, M. Valkonen, K. Kartasalo, L. Latonen, P. Ruusuvuori, K. Liimatainen, S. Albarqouni, B. Mungal, A.
George, S. Demirci, N. Navab, S. Watanabe, S. Seno, Y. Takenaka, H. Matsuda, H.A. Phoulady, V. Kovalev, A. Kalinovsky, V. Liauchuk, G. Bueno, M.M. Fernandez-Carrobles, I. Serrano, O. Deniz, D. Racoceanu, R. Venâncio, Diagnostic assessment of deep learning algorithms for detection of lymph node metastases in women with breast cancer. JAMA 318, 2199-2210 (2017)

19. G. Litjens, C.I. Sánchez, N. Timofeeva, M. Hermsen, I. Nagtegaal, I. Kovacs, C. Hulsbergen-Van De, P. Kaa, B.v.G. Bult, J.A.W.M. van der Laak, Deep learning as a tool for increased accuracy and efficiency of histopathological diagnosis. Sci. Rep. 6, 26286 (2016)

20. F. Ciompi, O.G.F. Geessink, B.E. Bejnordi, G.S. De Souza, A. Baidoshvili, G.J.S. Litjens, B. van Ginneken, I.D. Nagtegaal, J.A.W.M. van der Laak, The importance of stain normalization in colorectal tissue classification with convolutional networks. Proc IEEE Int. Symp. Biomed. Imaging 14, 160-163 (2017)

21. B. Ehteshami Bejnordi, G. Litjens, N. Timofeeva, I. Otte-Höller, A. Homeyer, N. Karssemeijer, J. van der Laak, Stain specific standardization of whole-slide histopathological images. IEEE Trans. Med. Imaging 35, 404-415 (2016)

22. QUASAR Collaborative Group, Adjuvant chemotherapy versus observation in patients with colorectal cancer: A randomised study. Lancet 370, 2020-2029 (2007)

23. R. Glynne-Jones, L. Wyrwicz, E. Tiret, G. Brown, C. Rödel, A. Cervantes, D. Arnold, Rectal cancer: ESMO clinical practice guidelines for diagnosis, treatment and follow-up. Ann. Oncol. 28, iv22iv40 (2017)

24. G.W. van Pelt, T.P. Sandberg, H. Morreau, H. Gelderblom, J.H.J.M. van Krieken, R.A.E.M. Tollenaar, W.E. Mesker, The tumourstroma ratio in colon cancer: The biological role and its prognostic impact. Histopathology 73, 197-206 (2018)

25. M. A. Smit, G. W. van Pelt, R. A. E. M. Tollenaar, W. E. Mesker, Uniform Noting for International application of the Tumour-stroma ratio as Easy Diagnostic tool (UNITED) study website. Dept. of surgery, Leiden University Medical Center (LUMC). http:// watchstroma.com/the-stroma-research/. Accessed 28 November 2018 